\title{
BIAS DRIFT ESTIMATION FOR MEMS GYROSCOPE USED IN INERTIAL NAVIGATION
}

\author{
Radosław CECHOWICZ \\ *Department of Automation, Faculty of Mechanical Engineering, Lublin University of Technology, \\ ul. Nadbystrzycka 36, 20-618 Lublin, Poland \\ r.cechowicz@pollub.pl
}

received 8 May 2016, revised 10 May 2017, accepted 15 May 2017

\begin{abstract}
MEMS gyroscopes can provide useful information for dead-reckoning navigation systems if suitable error compensation algorithm is applied. If there is information from other sources available, usually the Kalman filter is used for this task. This work focuses on improving the performance of the sensor if no other information is available and the integration error should be kept low during periods of still (no movement) operation. A filtering algorithm is proposed to follow bias change during sensor operation to reduce integration error and extend time between successive sensor calibrations. The advantage of the proposed solution is its low computational complexity which allows implementing it directly in the micro-controller of controlling the MEMS gyroscope. An intelligent sensor can be build this way, suitable for use in control systems for mobile platforms. Presented results of a simple experiment show the improvement of the angle estimation. During the 12 hours experiment with a common MEMS sensor and no thermal compensation, the maximum orientation angle error was below 8 degrees.
\end{abstract}

Key words: MEMS Gyroscope, Inertial Navigation, Mobile Platform, Bias Estimation, Angle Of Orientation, Filtering, Gauss Distribution

\section{INTRODUCTION}

One of the barriers to the development of mobile platforms designed to work in industrial halls and public buildings are the difficulties in identifying the position of the platform (in relation to the other elements of the environment/building). In the AGV systems operating in warehouses, this problem is usually solved by installing hardware (fixed) routes or way-points. Depending on the chosen technology, these paths can be marked with contrasting paint, magnetic stripe, inductive wires, or by surface modifications (guiding canals, kerbs, etc.). Orientation in space can then be kept by reading or counting route markings - for example bar codes, QR codes, RFID tokens, magnetic strips, etc. In some systems, the position of the moving object, in relation to fixed or passive beacons, can be determined by triangulation (ljaz et al., 2013; Mautz, 2009). Selected industrial control systems for autonomous vehicles have been documented for example in Ganesharajah et al. (1988).

Such navigation systems share a common problem - the limited flexibility of the path forces users to follow fixed routes (or go over fixed way-points). In consequence, some space is reserved for the exclusive use of the transport system. Any change of the route can only be done by installing new markings and reserving new space for the AGV system (Herrero-Perez et al., 2014). Since all routes have to be marked, the infrastructure must cover the entire area where the automatic transport system is used.

If the autonomy of transport system users (mobile platforms) were increased by (partial or total) detaching them from permanently marked routes, it would allow:

- to increase the flexibility of the system allowing adjusting the transport routes to the dynamic change of the environment (e.g. the emergence of obstacles, temporary route closure, etc.).
- to reduce the number of fixed way-points decreasing the cost of the infrastructure.

Autonomous mobile platforms must be equipped with a navigation system that provides the ability to move independently in areas where the observation of way-points is not possible. There, the on-board controller must navigate using the data from the local sensors (dead-reckoning).

Partial autonomy of the mobile platform can be achieved using the existing technologies. For example, in Lee and Yang (2012) a Hall sensor was used to measure deviations from the trajectory while passing over magnetic way-points. Similarly, using QR codes and a camera mounted on the moving platform, the deviation from the track is measured in Kiva robots (Guizzo, 2008; Mountz, 2005). Local control system operating in these robots identifies the properties of the platform (like yaw factor) on the basis of measurements from multiple on-board sensors and the observation of fixed reference points. As a result, robots can move autonomously through the distance between reference points.

Authors of the article (Acosta Calderon et al., 2015) proposed a solution that allowed removing all hardware way-points. In this system, the platform "learns" the route at the time of the first run (which is done in manual control mode) by saving data from the on-board sensors. After that, the platform can follow the same route autonomously, using the previously collected data. To change the route, it is necessary to either guide the robot through the new path, or to download an existing map from the robot that had already followed it.

Similar solution, using data from encoders and from the laser scanner, is described in Herrero_Perez et al. (2013). The advantage of the solutions of this kind is that the platform is able to perform some operations without reference to the fixed infrastructure points. Thus, platform route can be easily adopted to the 
current configuration of machines, pallets and obstacles in the hall.

Dead-reckoning navigation systems have been known and used for many years in maritime navigation, aviation and military. It can also be used to support the automotive GPS systems in areas where the signal is disrupted or unavailable (Enberg, 2015; Fang et al., 2005). Dead-reckoning is possible on the basis of data from inertial sensors - accelerometers and gyroscopes. Successful applications, make use mainly of high-quality gyroscopes, with low random noise and high durability. However, cheaper micro-electromechanical (MEMS) devices are increasingly popular. In Enberg (2015) it was shown that useful navigational information can be obtained by combining inertial sensors and incremental encoders.

Inertial sensors are used for navigating within buildings. One of the reported (Harle, 2013; Yuan and Chen, 2014; Zhang et al., 2013) applications is the use of an accelerometer and a gyroscope from the mobile phone to determine the position of the man inside the building. Position is calculated here by measuring the length and direction of steps. Detection of steps allows splitting the signal from sensors to small pieces, each a few seconds long (corresponding to a single step). This allows eliminating errors caused by the instability of inertial sensors and greatly improves the measurement. This works properly even with low-grade sensors (Scarlett, 2007). The system allows navigating in 3D, so it is possible to determine where and on which floor the owner of the phone is currently located (Fuchs et al., 2011), and use this information in a personalized guide or security system. A similar method was used in Gersdorf and Freese (2013), to estimate the position of a wheelchair using the signal from inertial sensors mounted on one of the wheels. The achieved accuracy of the measurement allowed practical use of the system. A similar system, described in Hedberg and Hammar (2015), was used to track the position of the train on the route between the stations.

\section{RESEARCH PROBLEM}

The problem shown here is a part of the larger project aiming to develop a hybrid navigation system for industrial mobile platforms working in a dynamic environment (typically inside a hall). Typical route of the mobile platform will consist of straight sections connected by arcs of small radius. The platform, equipped with a collision-preventing control system, will use dead-reckoning to navigate between scattered reference points. The reference points installed in the hall, will provide the way to accurately determine the position and orientation of the platform according to the fixed coordinate system. However, this will be possible only in a few selected areas. Inside the hall, the platform will move on a smooth, levelled surface, but it will have the ability to leave the hall and go on an outside lot where the surface will not be smooth (e.g. paved with cobbles).

A micro-electromechanical gyroscope mounted on the platform will be one of the elements of the navigation system. It will be used to measure the angle of rotation during turns, and to detect changes in the orientation of the platform caused by external factors (slip, user/operator actions, collisions with stationary or moving objects, etc.). The gyroscope system should be able to properly estimate the orientation of the platform between successive corrections made in the areas where the reference points were accessible for measurement. The time between successive adjustments is not fixed and can vary from a few seconds to several hours, depending on the type of task to be performed by the platform.

Because of the planned operating mode, the significant feature of the gyroscopic system should be the ability to maintain small orientation errors during longer stops outside the correction areas. It would allow to decrease the density of fixed position measurement points and enable the platform to go outside the platform to go outside the area of normal operation.

The research described in the article was conducted on a test rig consisting of a SCARA robot placed on a fixed platform.

\subsection{Test Rig}

The research described in the article was conducted on a test rig consisting of a SCARA robot placed on a fixed platform. MEMS sensor (gyroscope) was attached to the robot arm, as shown in Fig. 1 The robot arm was chosen instead of a mobile platform as it allowed to obtain accurate measurements of the actual angle of orientation and simulate driving on an arc of specified radius with precisely controlled angular velocity. The robot arm orientation was monitored during the tests with the rate of 10 measurements per second.

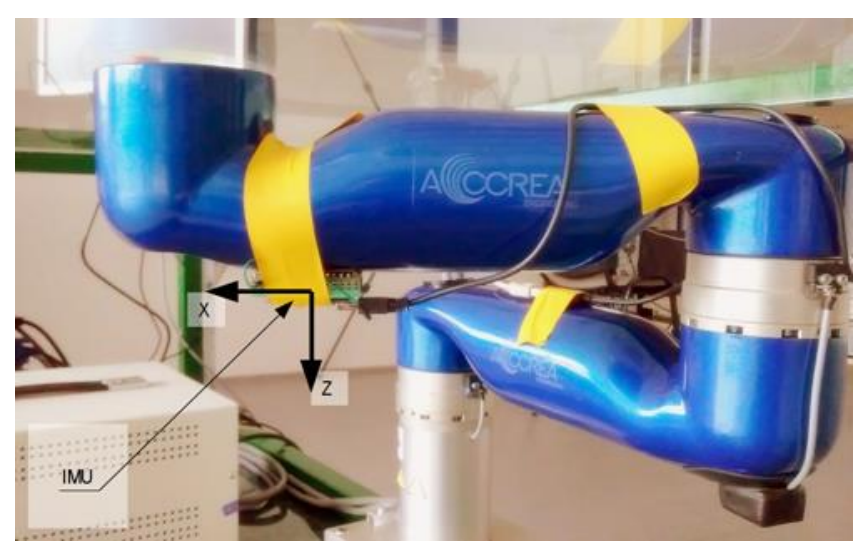

Fig. 1. The test rig with a gyroscopic sensor

\subsection{The Gyroscope}

The L3GD20H (ST Microelectronics) micro-electromechanical gyroscope was used during the tests. It is intended to be used in augmented reality, automation, robotics and navigation. The package comprises three gyroscopes measuring the rotational speed about axes of the Cartesian system. The basic technical data of the gyroscope are shown in Tab. 1.

Tab. 1. Basic technical data of the MEMS gyro (Fuchs et al., 2011)

\begin{tabular}{|c|c|c|c|}
\hline Gyro range & $+/-245 \mathrm{dps}$ & Resolution & $0.00875 \mathrm{dps} / \mathrm{LSB}$ \\
\hline Zero rate bias & $\begin{array}{c}+/-25 \mathrm{dps} \\
(@ 2000 \mathrm{dps})\end{array}$ & $\begin{array}{c}\text { Rate noise } \\
\text { density }\end{array}$ & $0.011 \mathrm{dps} / \mathrm{Hz}^{1 / 2}$ \\
\hline $\begin{array}{c}\text { Angle Random } \\
\text { Walk }\end{array}$ & $0.66 \% \mathrm{~h}^{1 / 2}$ & $\begin{array}{c}\text { Digital output } \\
\text { data rate }\end{array}$ & $378.8 \mathrm{~Hz}$ \\
\hline $\begin{array}{c}\text { Temperature } \\
\text { sensitivity change }\end{array}$ & $2 \%$ & $\begin{array}{c}\text { Temp. zero } \\
\text { rate change }\end{array}$ & $+/-0.04 \mathrm{dps} /{ }^{\circ} \mathrm{C}$ \\
\hline
\end{tabular}

Data from all three axes will be used in the navigation system. 
For the measurement of the angle of orientation of the platform moving on a horizontal surface, the most important information will come from the $Z$ axis, perpendicular to the plane of movement. Further, in this article, the results connected to rotation about $Z$ axis are presented.

\subsection{Evaluation of the quality of the gyro sensor}

The standard method for assessing the quality of the gyro is the Allan test (Institute of Electrical and Electronics Engineers, 2004). The test shall be carried out in a static state - gyroscope remains stationary for the duration of the test. Analysis of the recorded data allows determining natural variability of the sensor and evaluating the stability of the signal. The Allan variance method is used to compare the quality of the gyroscopes and to assess the nature of the signal disturbances. The methodology of the test is documented in Part C of the IEEE951 standard (Allan, 1966). Methods of testing and evaluation of oscillatory gyroscopes (MEMS) have been described in [24]], in the IEEE 1431 standard (Thielman et al., 2002), and in the IEC 62047-20.

The result of the Allan test for the $Z$ axis gyro tested is shown in Fig. 2. The chart shows that the main source of noise in the range of sampling times from $0.01 \mathrm{~s}$ to $100 \mathrm{~s}$ is the angle random walk (ARW), which is approximately $0.0110 / \mathrm{s} / \mathrm{Hz}^{1 / 2}$. Stability of the bias for the axis $\mathrm{Z}$ is $3.58 \% \mathrm{~h}$. Based on these results, the gyroscope can be included in the class of industrial equipment. However, there is a spread of quality in MEMS gyroscopes. Even the units from the same housing can have different properties. For example, axes $X$ and $Y$ from the chip used in the tests had shown the ARW about $0.1 \mathrm{dps} / \mathrm{Hz}^{1 / 2}$. Measurements made on other units of the same type also gave different results.

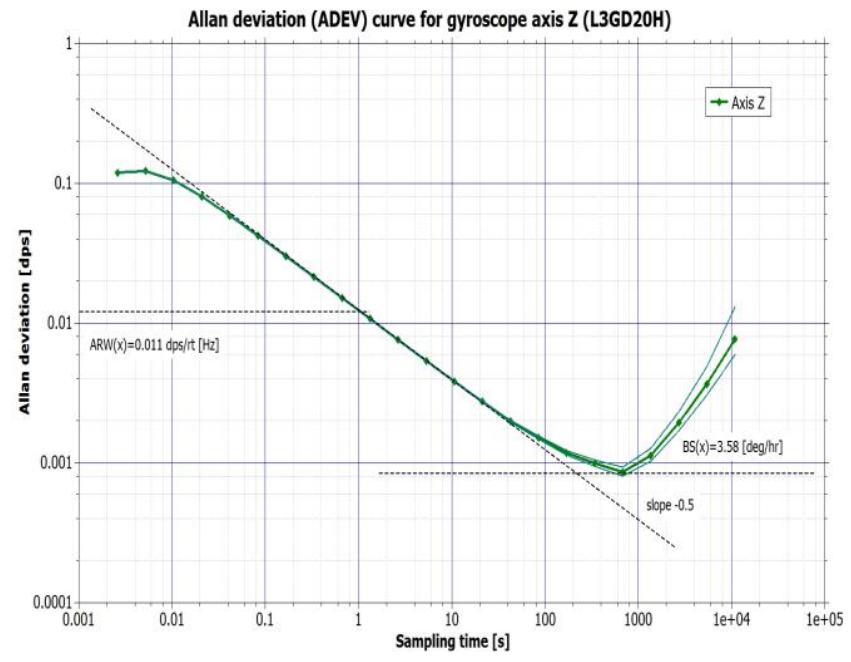

Fig. 2. Allan variance curve for the $Z$ axis gyro $L 3 G D 20 H$

The instability of the bias and the angle random walk are the two main sources of gyroscope error. Both factors are random, and their effect usually limits the practical application of individual sensors to applications that do not require observation time (measurements) longer than a few seconds. Random processes occurring in MEMS gyroscopes limit their ability to detect low rotational speeds and make it difficult for the control systems to correctly interpret the signal under static conditions.
Identification of error sources and development of a model of the sensor may be one of the methods to achieve a system suitable for the practical use in navigation. The method is used mainly for high-end sensors, whose parameters are stable over time. In the case of MEMS, it is possible to develop a model (Enberg, 2015), but, as shown in Barett (2014), it does not always lead to a significant reduction in measurement error.

The mechanical properties of MEMS sensors can also be an important factor affecting the accuracy (Weinberg, 2011) of measurements. The measurements from a sensor with weak mechanical properties (e.g. with a tendency to resonate) exposed to vibrations may show much greater error than predicted in technical data sheets.

Improvement in accuracy can be obtained using the technique of associating data from several sensors (sensor fusion). Among the solutions described in the literature, the following can be cited: measuring the rotational speed by means of appropriately arranged accelerometers (Chatterjee et al., 2015), averaging measurement of several gyroscopes forming a matrix (Jiang et al., 2013), using the information about the state of the object to find the most likely estimate (Herrero-Perez et al., 2013; Romaniuk and Gosiewski, 2014).

Another method to improve the accuracy of the gyroscope may be tracking of the actual value of the bias. Since bias instability is one of main factors contributing to gyroscope error, finding a way to estimate it could improve the quality of angle estimation.. One of such approaches, using extended Kalman filter, is shown in Hyyti and Visala (2015).

\subsection{Characteristics of the gyroscope signal}

For an ideal gyro sensor, angular velocity $w(t)$ of the platform at the moment $t$ is proportional to the signal $x(t)$ from the sensor after subtracting the bias $\mathrm{x}_{\mathrm{b}}(\mathrm{t})$ :

$\omega(t)=R\left(x(t)-x_{b}(t)\right)$

where: $\mathrm{R}$ - coefficient of proportionality.

The expected value of the bias $x_{b}$ is specified in the technical data (ZeroRateBias).

Tab. 2. Descriptive statistics for the gyro signal (sample length 6s)

\begin{tabular}{|c|r|c|r|}
\hline Parameter & $\begin{array}{c}\text { Measure- } \\
\text { ment(X) }\end{array}$ & Parameter & $\begin{array}{c}\text { Measure- } \\
\text { ment(X) }\end{array}$ \\
\hline Minimum value & -100 & Average $\mathbf{X s r}_{\mathbf{s r}}$ & $\mathbf{- 5 2}$ \\
\hline First quartile & -58 & Std. Deviation $\boldsymbol{\sigma}$ & 11 \\
\hline Median & -51 & Skewness & 0 \\
\hline Third quartile & -44 & Kurtosis & 4 \\
\hline Maximum value & -9 & & \\
\hline
\end{tabular}

The actual MEMS gyro signal is random. If the platform is stationary, and the recording time does not exceed a few dozen seconds, the signal from the MEMS gyro sensor can be approximated with a Gaussian distribution with the central value $\mathrm{x}_{\text {śr }}$ and the deviation $\sigma$. The descriptive statistics of the $6 \mathrm{~s}$ sample of the stationary gyroscope signal is shown in Tab. 2. Fig. 3 shows the histogram of this signal and the Gaussian distribution with parameters $w_{\text {śr }}$ and $\sigma$ from Tab. 2. The random nature of the signal can 
also be shown with the probability plot (Fig. 4) and the result of the Allan analysis (Fig. 2).

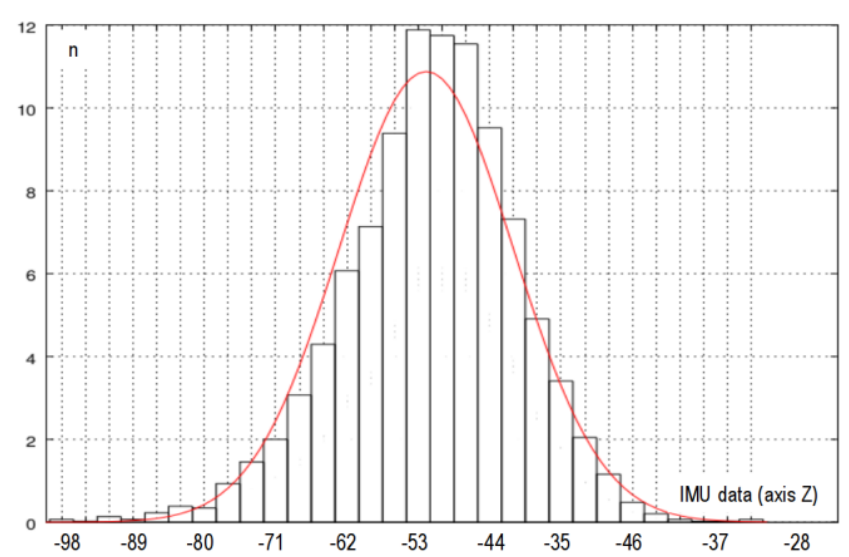

Fig. 3. A histogram signal from the $Z$ axis gyro and a normal distribution with the parameters calculated from the measurements (Tab. 2)

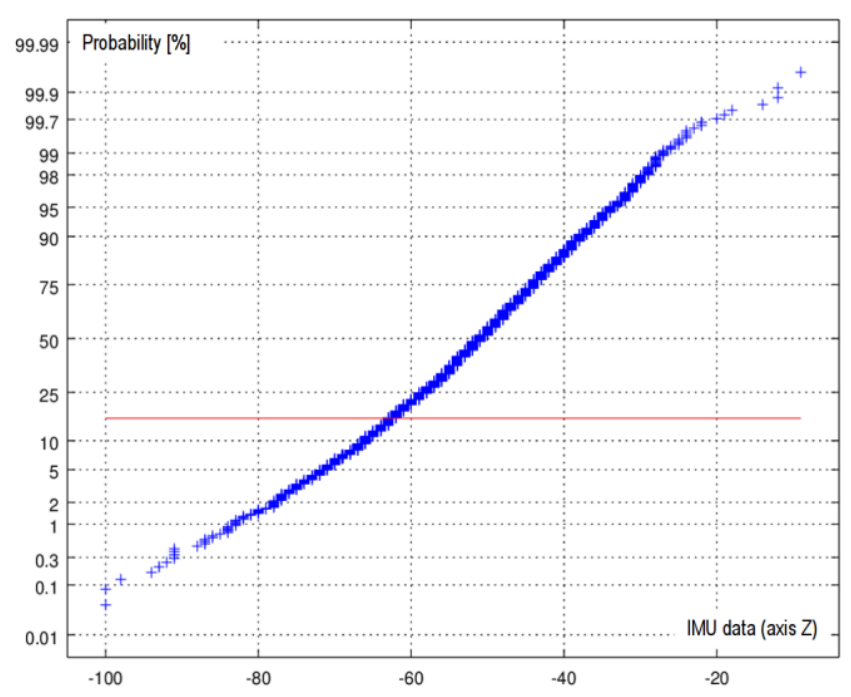

Fig. 4. Probability plot for the signal from the gyro Z-axis

The length of sampling time over which the central value of the Gaussian distribution can be computed, can be determined from the Allan variance curve. For the tested sensor that sampling time is from $0.1 \mathrm{~s}$ to tens of seconds, since in this range the error from bias instability is not yet decisive.

The noise to signal ratio determines the accuracy of the rotational speed measurement. For the test sensor, the noise amplitude $(6 \sigma=66)$ is only $0.2 \%$ of the measurement range. In applications where the rotational speed rather than orientation is measured (e.g. gesture detection), this accuracy is usually sufficient and this error can be ignored.

The average rotational speed of the platform during the period $\Delta t_{o}$ can be determined from the formula:

$\omega_{s r}\left(\Delta t_{o}\right)=R\left(x_{s r}\left(\Delta t_{o}\right)-x_{b}\left(\Delta t_{o}\right)\right)$

If the platform remained still during this time that is if $\omega_{s r}\left(\Delta t_{o}\right)=0$, then:

$x_{b}\left(\Delta t_{o}\right)=x_{\text {sr }}\left(\Delta t_{o}\right)$

The value of bias can therefore be found by computing the mean value of signal $x(t)$ at the time when the platform remains stationary. The maximum averaging time is limited by the bias instability and the working conditions of the navigation system (length and frequency of stops).

In Fig. 5 the signal from the gyro is divided into uniform segments of 3 seconds. Since the actual sampling frequency was $384 \mathrm{~Hz}$, there were 1,150 samples in each segment. Then, descriptive statistics was calculated for each segment, as in Tab. 2. On Fig. 5, there are shown: the average value in each segment, the standard deviation, the first and third quartile. Points which are outside the three quartile range (outliers) are marked with circles.

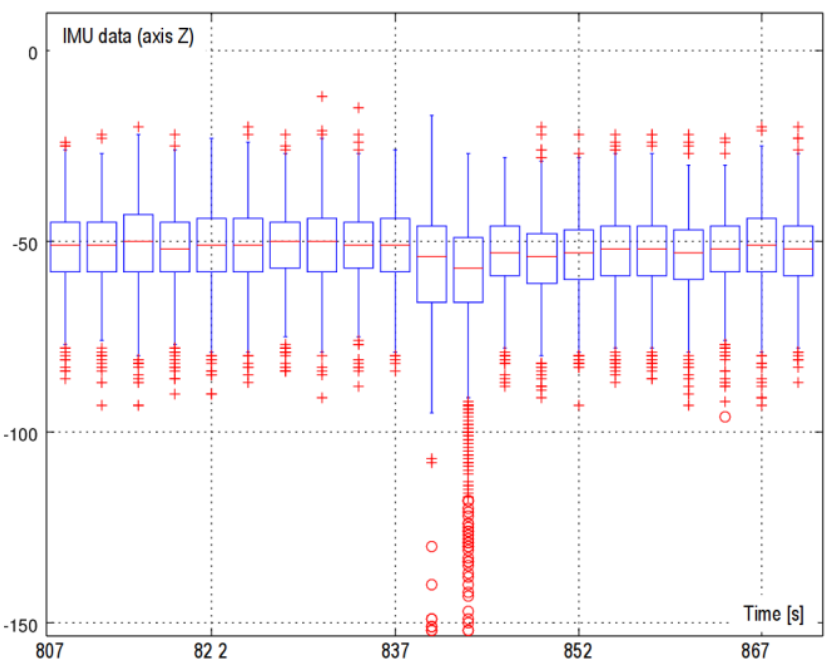

Fig. 5. Change of the gyro signal while in motion

In the middle of the time shown in Fig. 5 (the time from 840 to 846 seconds) the platform with a sensor made rotation of the angle $\Theta=220^{\circ}$, which lasted about 3 seconds. Two data segments which contain the rotation data are easy to distinguish from the rest. They have a significantly higher dispersion (range) and a slightly shifted average. The range of the segments is increased because the rotational speed of the platform, and the signal from the gyroscope changed proportionally. The range of the segments recorded during the constant speed motion will also be slightly larger than the stationary range due to unevenness of speed and an increased level of noise while driving (vibration, uneven ground etc.). The range of segments can therefore be used as a criterion for the selection of episodes in which the platform remained stationary.

The length of the segment (averaging time) will be primarily chosen with regard to technical reasons. Increasing the length of the segment up to several dozens of seconds, improves the sensitivity of the range criterion - it will then be possible to detect even small changes in the gyro signal (and low rotational speeds). However, this also extends the time required to register at least one segment representing pure stationary state, which may limit the usefulness of the system.

Bias $\left(x_{b}\right)$ changes with time. This is the cause of the so-called "flow" or "drift" of the gyro readings which in some applications makes it necessary to periodically calibrate the gyro. The phenomenon of drift causes also a sharp increase in the orientation angle error, which is calculated by integrating the gyro signal over time. Since the MEMS gyroscopes exhibit relatively large drift they are usually not suitable for direct use in navigation.

Allan graph (Fig. 2) shows that the bias instability rate of the test sensor is $\mathrm{d} \omega_{o}=3.58 \% / \mathrm{h}$. In addition, temperature error of the 
sensor may reach $\mathrm{d} \omega_{T}=+/-0.005 \mathrm{dps} /{ }^{\circ} \mathrm{C}=+/-180 / \mathrm{h} /{ }^{\circ} \mathrm{C}$. Even in stable operating conditions (temperature stabilization to ${ }^{\circ} \mathrm{C}$, no mechanical vibrations) it could mean that the orientation angle error after 1 hour observation will be greater than $20^{\circ}$.

\subsection{Estimation of the bias change}

Let us suppose that at the moment $\mathrm{t} 1$ the angle of orientation of the platform was $\Theta_{1}$. The angle of orientation of the platform $\Theta_{n}$ at the moment $t_{n}$ is calculated by integrating the speed signal:

$\Theta_{n}=\Theta_{1}+\frac{1}{f_{s}} \sum_{i=1}^{n} \omega_{z}\left(t_{i}\right) \Theta_{n}=\Theta_{1}+\frac{R}{f_{s}} \sum_{i=1}^{n} x\left(t_{i}\right)-x_{b}\left(t_{i}\right)$

where: $f_{s}$ - sampling rate (here $384 \mathrm{~Hz}$ )

To determine the correct angle, it is necessary to know the value of the bias $x_{b}$.

Observing the signal of stationary gyroscope, one can notice that the speed of bias change is slow (at a rate of ten degrees per hour) compared to the change caused by rotation of a typical mobile platform (a few degrees per second). These two processes can be distinguished from each other by observing a signal from the sensor. To make this possible in a statistical sense, an assumption should be made that the nature of the distribution and the standard deviation of the signal generated by the stationary sensor remains unchanged over time (the natural process distribution does not change). Only the central value of the distribution $\mathrm{x}_{\text {Wed }}$ changes over time during the operation of the sensor.

Tracking the central value $\mathrm{x}_{\text {śr }}$ (bias) changes is easy when the platform remains stationary. The problem is to find the correct value of bias when the platform is moving. As it is difficult to determine bias value while the platform is rotating, all segments recorded during the motion should be discarded using the range criterion.

In the experiment described below the following criterion was used to discard segments recorded during platform movement:

$R x<7 \sigma_{\text {śr }}$

where: $R x$ - range of the segment, $\sigma_{\dot{s} r} \sigma_{\dot{s} r}$ - average standard deviation of the segments recorded in stationary conditions.

To detect slow changes in the average value (bias) and separate them from noise, a further selection step was proposed, wherein the average values of segments were compared against the moving average of some preceding segments. At this stage, all the segments with averages outside the typical area of variability were discarded. Typical area of variability of normal distribution is:

$\Delta x=\left[x_{\text {śr }}-\sigma, x_{\text {śr }}+\sigma\right]$

So, all the segments with average outside the limits were discarded:

$x_{\dot{s} r}\left(t_{i}\right)>\frac{1}{n} \sum_{i-n}^{i} x_{\dot{s} r}\left(t_{k}\right)-\sigma_{\text {śr }}$

$x_{\text {śr }}\left(t_{i}\right)<\frac{1}{n} \sum_{i-n}^{i} x_{\text {śr }}\left(t_{k}\right)+\sigma_{\text {śr }}$

where: $x_{\text {śr }}\left(t_{i}\right) \sigma_{\text {śr }}$ mean value of the segment, $n \sigma_{\text {śr }}$ number of previously recorded segments to be used for the moving average. During the experiment, the value of $n$ was chosen arbitrary.

\subsection{Obtained results}

Fig. 6 and Fig. 7 show the results of the experiment involving the measurement of the orientation angle of the platform rotating about 0.2 radians every 6 minutes. The experiment lasted 12 hours and its purpose was to check the stability of the MEMS gyroscope and to measure the orientation error. The platform was moving automatically, according to a pre-programmed sequence. The actual angle of orientation was measured with incremental encoders (with accuracy 0.010).

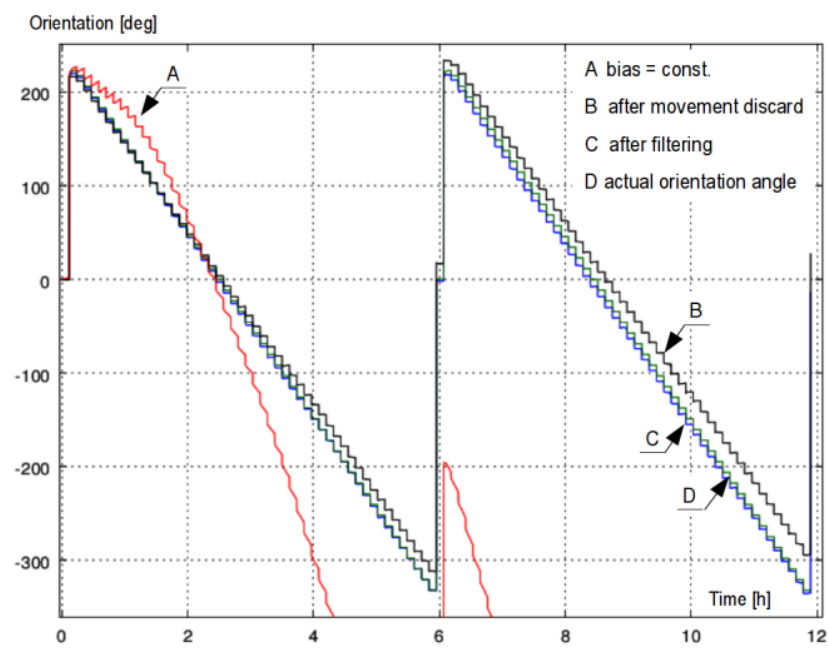

Fig. 6. The result of the experiment described in section 2.6

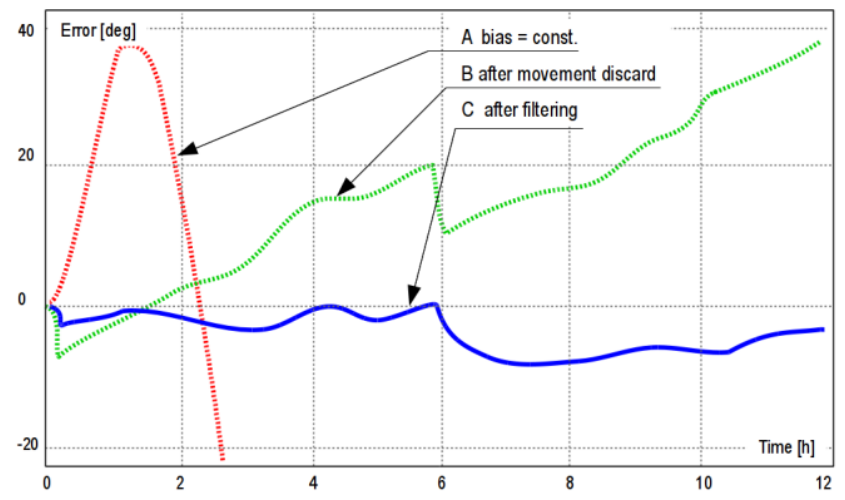

Fig. 7. Comparison of the angle of orientation errors

The angle of orientation has been determined by three different methods:

A - the bias xp appointed at the beginning of the experiment, remained unchanged for the rest of the time.

$B$ - the bias was determined throughout the experiment, from segments remaining after the application of the criterion (6)

$\mathrm{C}$ - the bias was determined from the segments that met criteria (5) and (7).

The actual angle of orientation of the platform is marked in Fig. 6 with the letter $D$.

Orientation angle errors are shown in Fig. 7. Orientation error was obtained according to the formula:

$E\left(t_{i}\right)=\Theta_{a}\left(t_{i}\right)-\Theta_{x}\left(t_{i}\right)$

where: $E(t)$ - orientation error, $\Theta_{a}\left(t_{i}\right)$ - the actual orientation 
angle of the platform at time $t, \Theta_{x}\left(t_{i}\right)$ - orientation angle is determined from the signal of the gyroscope

In the case of the method $A$, the orientation error exceeded 200 after a few minutes. Then, the absolute value of the angle grew at a rate of about $40^{\circ}$ per hour. The change of the direction of the error accumulation was due to thermal phenomena - the temperature of the gyro stabilized after about 4 hours after the experiment was started (the MEMS unit was mounted directly on a PCB, without any heat shield)

Using the range criterion (5) for segment selection and determining the offset value dynamically at standstill helped to reduce the rate error to about $5^{\circ}$ per hour. Due to the insufficient sensitivity of this method, the error is growing rapidly during the movements performed at low speed (mainly at final stages of the platform movement).

An additional selection criterion using the typical area of variability of the signal (7) allowed reducing the average rate of change of error to less than 10 per hour. This method still has too low sensitivity to be able to detect low-speed movement but the improvement in performance is clearly visible.

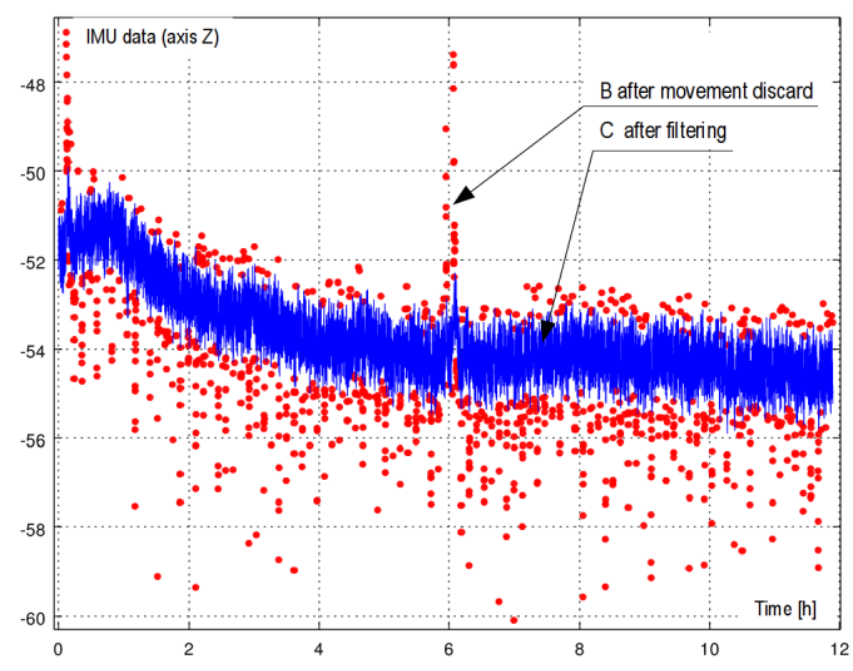

Fig. 8. Estimated bias value over time. The points rejected in the second selection phase are marked with red dots

Fig. 8 shows the estimated bias value during the experiment. Red dots mark the points that passed the first criterion (5). The blue line marks the points that additionally passed the criterion (7).

It is apparent (in Fig. 8) that the change of bias is not linear. The sensor temperature is one of the factors that greatly affect the measurement. It is difficult to identify and quantize the share of other factors.

\section{CONCLUSION}

The advantage of the described algorithm is its low computational complexity which allows implementing it directly in the micro-controller that provides communication with the MEMS gyroscope. An intelligent sensor can be build this way, suitable for use in control systems for mobile platforms, or other objects which do not rotate continuously.

During the experiment the thermal compensation of the gyroscope was not used. The method presented allows detecting any shifts of bias in the gyro signal - including those caused by the temperature change.
Two gyro sensors of the same type were used. In both cases, similar results were obtained.

A disadvantage of the proposed method is poor detection of low speed rotations. As a result, the sensor is not suitable for detecting deviation from the straight path or slow changes of the orientation of the platform. These must be detected by other methods.

An important limitation of the proposed method the necessity to stop the platform regularly in order to estimate the average value of the standstill signal and compute the current bias value. However, the typical work pattern of a warehouse robot or industrial AGV contains frequent stops for loading/unloading and maintenance operations so this should not be a problem.

Gyroscopes and all dead-reckoning systems work in incremental mode which is prone to error build-up over time. To extend the time between successive calibrations of the sensor, either a better sensor must be fitted or the measurement procedure must be improved.

Despite the drawbacks, the proposed method allows increasing the accuracy of the MEMS gyro sufficiently enough to use it as a secondary device for navigation in short distances.

Further research will focus on better detection of stationary state, possibly delegating some computational workload to a more powerful processor in the navigation control system.

\section{REFERENCES}

1. Acosta Calderon C.A., Mohan E.R., Ng B.S. (2015), Development of a hospital mobile platform for logistics tasks, Digital Communications and Networks, 1 (2), 102-111.

2. Allan D.W. (1966), Statistics of atomic frequency standards, Proceedings of the IEEE, 54 (2), 221-230.

3. Barrett J.M. (2014), Analyzing and Modeling Low-Cost MEMS IMUs for use in an Inertial Navigation System, Worcester Polytechnic Institute.

4. Chatterjee G., Latorre L., Mailly F., Nouet P., Hachelef N., Oueda C. (2015), Smart-MEMS based inertial measurement units: gyro-free approach to improve the grade, Microsystem Technologies, $1-10$

5. Enberg D. (2015), Performance Evaluation of Short Time Dead Reckoning for Navigation of an Autonomous Vehicle, Department of Electrical Engineering, Linköpings universitet

6. Fang L., Antsaklis P.J., Montestruque L.A., McMickell M.B., Lemmon M., Sun Y., Fang H., Koutroulis I., Haenggi M., Xie M., Xie X. (2005) Design of a wireless assisted pedestrian dead reckoning system - the NavMote experience, IEEE Trans Instrum Meas, 54, 2342-2358

7. Ferraina M. (2015), L3GD20H: 3-axis digital output gyroscope STMicroelectronics, DocID026442 Rev 2

8. Fuchs C., Aschenbruck N., Martini P., Wieneke M (2011), Indoor tracking for mission critical scenarios: A survey, Pervasive and Mobile Computing, 7 (1), 1-15.

9. Ganesharajah T., Hall N.G., Sriskandarajah C. (1988), Design and operational issues in AGV-served manufacturing systems, Annals of Operations Research, 76 (0), 109-154.

10. Gersdorf B., Freese U. (2013), A Kalman Filter for Odometry using a Wheel Mounted Inertial Sensor, ICINCO, 1, 388-395.

11. Guizzo E. (2008), Three Engineers, Hundreds of Robots, One Warehouse, IEEE Spectrum, 45(7), 26-34.

12. Harle R. (2013), A Survey of Indoor Inertial Positioning Systems for Pedestrians, IEEE Communications Surveys \& Tutorials, 15(3), 1281-1293.

13. Hedberg E., Hammar M. (2015), Train Localization and Speed Estimation Using On-Board Inertial and Magnetic Sensors, Department of Electrical Engineering, Linköpings universitet 
14. Herrero-Perez D., Jose J., Martinez-Barbera H. (2013), An Accurate and Robust Flexible Guidance System for Indoor Industrial Environments, International Journal of Advanced Robotic Systems, 10 (1), $1-9$

15. Hyyti H., Visala A. (2015), A DCM Based Attitude Estimation Algorithm for Low-Cost MEMS IMUs, International Journal of Navigation \& Observation, 2015, 1-18.

16. Ijaz F., Yang H.K., Ahmad A.W., Lee C. (2013), Indoor positioning: A review of indoor ultrasonic positioning systems, Advanced Communication Technology (ICACT), 2013 15th International Conference, 1146-1150.

17. Institute of Electrical and Electronics Engineers (2004), IEEE standard specification format guide and test procedure for coriolis vibratory gyros, Institute of Electrical and Electronics Engineers, New York.

18. Jiang C., Xue L., Chang H., Yuan W. (2012), Signal Processing of MEMS Gyroscope Arrays to Improve Accuracy Using a 1st Order Markov for Rate Signal Modeling, Sensors, 12(12), 172-1737.

19. Lee S.-Y., Yang H.-W. (2012), Navigation of automated guided vehicles using magnet spot guidance method, Robotics and Computer-Integrated Manufacturing, 28(3), 425-436.

20. Mautz R. (2009), Overview of current indoor positioning systems, Geodesy and Cartography, 35(1), 18-22.

21. Mountz M.C. (2005), Material handling system and method using mobile autonomous inventory trays and peer-to-peer communications, US/6950722

22. Romaniuk S. Gosiewski Z. (2014), Kalman Filter Realization for Orientation and Position Estimation on Dedicated Processor, Acta Mechanica et Automatica, 8(2), 88-94

23. Scarlett J. (2007), Enhancing the performance of pedometers using a single accelerometer, Application Note, Analog Devices, AN-900

24. STMicroelectronics (2013), MEMS motion sensor: three-axis digital output gyroscope L3GD2OH Datasheet.

25. Thielman L.O., Bennett S., Barker C.H., Ash M.E. (2002), Proposed IEEE Coriolis Vibratory Gyro standard and other inertial sensor standards, Position Location and Navigation Symposium, 2002 IEEE, 351-358.

26. Weinberg H. (2011), Gyro mechanical performance: The most important parameter, Technical Article MS-2158, Analog Devices

27. Yuan Q., Chen I.-M. (2014), Localization and velocity tracking of human via 3 IMU sensors, Sensors \& Actuators: A. Physical, 212, 25-33.

28. Zhang R., Bannoura A., Hoflinger F., Reindl L.M., Schindelhauer C. (2013), Indoor localization using a smart phone, Sensors Applications Symposium (SAS), 2013 IEEE, 38-42. 